\title{
Fan Sub-Equation method with Improved Algorithms for Travelling Wave Solutions of Jimbo-Miwa Equation
}

\author{
Sheng Zhang ${ }^{1, a}$ and Aoxue Peng ${ }^{1, b}$ \\ ${ }^{1}$ School of Mathematics and Physics, Bohai University, Jinzhou 121013, PR China \\ aszhangchina@126.com, b382337849@qq.com
}

Keywords: Fan sub-equation method; Travelling wave solution; Jimbo-Miwa equation

\begin{abstract}
In this paper, the (3+1)-dimensional Jimbo-Miwa equation is solved by Fan sub-equation method with improved algorithms. As a result, many new and more general travelling wave solutions are obtained including kink-shaped soliton solutions, rational solutions, triangular periodic solutions, Jacobi and Weierstrass doubly periodic wave solutions. At a certain limit condition, the obtained Jacobi elliptic periodic wave solutions can degenerate into soliton solutions. It is shown that the improved algorithms of Fan sub-equation method can lead to such solutions with external linear functions possessing two remarkable evolutionary properties: (i) the wave propagation is skew; (ii) the amplitude enlarges along with the increasing time.
\end{abstract}

\section{Introduction}

With the development of computer science, some symbolic computation systems like Mathematica or Maple have been used to perform the complex and tedious computation on computers for constructing exact solutions of nonlinear evolution equations (NLEEs), such as those in [1-10]. Searching for exact travelling wave solutions of NLEEs plays an important role in the study of nonlinear physical phenomena. In 2003, the so-called Fan sub-equation method [11] was proposed for solving NLEEs and received many applications [12-14]. Recently, Zhang and Peng [15] improved Fan sub-equation by modifying its algorithms. One of the advantages of this improved algorithms can lead to such solutions with external linear functions of some given NLEEs. This present paper is motivated by the desire to show the effectiveness and advantage of the improved algorithms [15] through the (3+1)-dimensional Jimbo-Miwa equation [9]:

$$
u_{x x x y}+3 u_{y} u_{x x}+3 u_{x} u_{x y}+2 u_{y t}-3 u_{x z}=0
$$

\section{Exact solutions}

In this section, we consider the (3+1)-dimensional Jimbo-Miwa equation (1). We take the following travelling wave transformation:

$u=u(x, y, z, t)=u(\xi), \quad \xi=a x+b y+c z-\omega t$,

where $a, b, c$ and $\omega$ are constants, then Eq. (1) is reduced into an ODE

$a^{3} b u^{(4)}+6 a^{2} b u^{\prime} u^{\prime \prime}-(3 a c+2 b \omega) u^{\prime \prime}=0$.

We then integrate Eq. (3) once with respect to $\xi$ and set the integration constant to zero, Eq. (3) becomes

$a^{3} b u^{(3)}+3 a^{2} b\left(u^{\prime}\right)^{2}-(3 a c+2 b \omega) u^{\prime}=0$.

Setting $u^{\prime}=v$, we have

$a^{3} b v^{\prime \prime}+3 a^{2} b v^{2}-(3 a c+2 b \omega) v=0$. 
According to the improved Fan sub-equation method [15] we suppose that Eq. (5) has the following formal solution

$$
v=\alpha_{2} \varphi^{2}(\xi)+\alpha_{1} \varphi(\xi)+\alpha_{0} .
$$

where $\varphi(\xi)$ satisfis a second-order linear ordinary differential equation (ODE):

$$
\varphi^{\prime 2}(\xi)=h_{0}+h_{1} \varphi(\xi)+h_{2} \varphi^{2}(\xi)+h_{3} \varphi^{3}(\xi)+h_{4} \varphi^{4}(\xi)
$$

Substituting Eq. (6) along with Eq. (7) into Eq. (5) and collecting all terms with the same order of $\varphi(\xi)$ together, then setting each coefficient of the polynomial to zero, we derive a set of algebraic equations for $a, b, c, \omega, \alpha_{0}, \alpha_{1}$ and $\alpha_{2}$ as follows:

$$
\begin{aligned}
\varphi^{\prime 2}(\xi)= & h_{0}+h_{1} \varphi(\xi)+h_{2} \varphi^{2}(\xi)+h_{3} \varphi^{3}(\xi)+h_{4} \varphi^{4}(\xi) . \\
\varphi^{0}(\xi): & a^{3} b \alpha_{1} h_{1}+4 a^{3} b \alpha_{2} h_{0}+6 a^{2} b \alpha_{0}^{2}-4 b \omega \alpha_{0}-6 a c \alpha_{0}=0, \\
\varphi^{1}(\xi): & a^{3} b \alpha_{1} h_{2}+3 a^{3} b \alpha_{2} h_{1}+6 a^{2} b \alpha_{1} \alpha_{2}-2 b \omega \alpha_{1}-3 a c \alpha_{1}=0, \\
\varphi^{2}(\xi): & 3 a^{3} b \alpha_{1} h_{3}+8 a^{3} b \alpha_{2} h_{2}+6 a^{2} b \alpha_{1}^{2}+12 a^{2} b \alpha_{0} \alpha_{2}-4 b \omega \alpha_{2}-6 a c \alpha_{2}=0, \\
\varphi^{3}(\xi): & 2 a^{3} b \alpha_{1} h_{4}+5 a^{3} b \alpha_{2} h_{3}+6 a^{2} b \alpha_{1} \alpha_{2}=0, \\
\varphi^{4}(\xi): & 2 a^{3} b \alpha_{2} h_{4}+a^{2} b \alpha_{2}^{2}=0 .
\end{aligned}
$$

With the help of Mathematica, from this set of algebraic equations we obtain five cases as follows:

Case 3.1, when $h_{3}=h_{1}=h_{0}=0$ :

$$
\begin{aligned}
& \alpha_{2}=-2 a h_{4}, \quad \alpha_{1}=0, \quad \alpha_{0}=0, \omega=\frac{-3 a c+4 a^{3} b h_{2}}{2 b}, \\
& \alpha_{2}=-2 a h_{4}, \quad \alpha_{1}=0, \quad \alpha_{0}=-\frac{4}{3} a h_{2}, \omega=\frac{-3 a c-4 a^{3} b h_{2}}{2 b} .
\end{aligned}
$$

We then obtain

$$
\begin{aligned}
& v=-2 a h_{4} \varphi^{2}(\xi), \quad \omega=\frac{-3 a c+4 a^{3} b h_{2}}{2 b}, \\
& v=-2 a h_{4} \varphi^{2}(\xi)-\frac{4}{3} a h_{2}, \omega=\frac{-3 a c-4 a^{3} b h_{2}}{2 b} .
\end{aligned}
$$

We substitute the general solutions [11] of Eq. (7) into Eqs. (16) and (17), respectively, and use Eq. (6), then three types of travelling wave solutions of Jimbo-Miwa equation (1) are obtained.

(i) If $h_{2}>0, h_{4}<0$, we obtain two kink-shaped soliton solutions

$$
u=2 a \sqrt{h_{2}} \tanh \left(\sqrt{h_{2}} \xi\right)+d_{1},
$$

where $\xi=a x+b y+c z+\frac{3 a c-4 a^{3} b h_{2}}{2 b} t$, here and hereafter $d_{1}$ is a arbitrary constant.

$$
u=2 a \sqrt{h_{2}} \tanh \left(\sqrt{h_{2}} \xi\right)-\frac{4}{3} a h_{2} \xi+d_{1}, \quad \xi=a x+b y+c z+\frac{3 a c+4 a^{3} b h_{2}}{2 b} t .
$$

(ii) If $h_{2}<0, h_{4}>0$, we obtain two triangular periodic solutions

$$
u=-2 a \sqrt{-h_{2}} \tan \left(\sqrt{-h_{2}} \xi\right)+d_{1}, \quad \xi=a x+b y+c z+\frac{3 a c-4 a^{3} b h_{2}}{2 b} t,
$$


$u=2 a \sqrt{-h_{2}} \tan \left(\sqrt{-h_{2}} \xi\right)-\frac{4}{3} a h_{2} \xi+d_{1}, \quad \xi=a x+b y+c z+\frac{3 a c+4 a^{3} b h_{2}}{2 b} t$.

(iii) If $h_{2}=0, h_{4}>0$, we obtain two rational solutions

$u=2 a \xi^{-1}+d_{1}, \quad \xi=a x+b y+c z+\frac{3 a c}{2 b} t$,

$u=2 a \xi^{-1}-\frac{4}{3} a h_{2} \xi+d_{1}, \quad \xi=a x+b y+c z+\frac{3 a c}{2 b} t$.

Case 3.2, when $h_{3}=h_{1}=0, h_{0}=\frac{h_{2}^{2}}{4 h_{4}}$ :

$\alpha_{2}=-2 a h_{4}, \quad \alpha_{1}=0, \quad \alpha_{0}=-a h_{2}, \omega=\frac{-3 a c-2 a^{3} b h_{2}}{2 b}$,

$\alpha_{2}=-2 a h_{4}, \quad \alpha_{1}=0, \alpha_{0}=-\frac{1}{3} a h_{2}, \omega=\frac{-3 a c+2 a^{3} b h_{2}}{2 b}$.

Therefore, we obtain

$v=-2 a h_{4} \varphi^{2}(\xi)-a h_{2}(\xi), \omega=\frac{-3 a c-2 a^{3} b h_{2}}{2 b}$,

$v=-2 a h_{4} \varphi^{2}(\xi)-\frac{1}{3} a h_{2}, \omega=\frac{-3 a c+2 a^{3} b h_{2}}{2 b}$.

We substitute the general solutions [11] of Eq. (7) into Eqs. (26) and (27), respectively, and use Eq. (6), then two types of travelling wave solutions of Jimbo-Miwa equation (1) are obtained.

(i) If $h_{2}<0, h_{4}<0$, we obtain two kink-shaped soliton solutions

$$
\begin{aligned}
& u=a \sqrt{-2 h_{2}} \tanh \left(\sqrt{-\frac{h_{2}}{2}} \xi\right)+d_{1}, \quad \xi=a x+b y+c z+\frac{3 a c+2 a^{3} b h_{2}}{2 b} t, \\
& u=a \sqrt{-2 h_{2}} \tanh \left(\sqrt{-\frac{h_{2}}{2}} \xi\right)+\frac{2}{3} a h_{2} \xi+d_{1}, \quad \xi=a x+b y+c z+\frac{3 a c-2 a^{3} b h_{2}}{2 b} t .
\end{aligned}
$$

(ii) If $h_{2}>0, h_{4}>0$, we obtain two triangular periodic solutions

$$
\begin{aligned}
& u=-2 a \sqrt{2 h_{2}} \tan \left(\sqrt{\frac{h_{2}}{2}} \xi\right)+a h_{2} \xi+d_{1}, \quad \xi=a x+b y+c z+\frac{3 a c+2 a^{3} b h_{2}}{2 b} t, \\
& u=-2 a \sqrt{2 h_{2}} \tan \left(\sqrt{\frac{h_{2}}{2}} \xi\right)+\frac{5}{3} a h_{2} \xi+d_{1}, \quad \xi=a x+b y+c z+\frac{3 a c-2 a^{3} b h_{2}}{2 b} t .
\end{aligned}
$$

Case 3.3, when $h_{3}=h_{1}=0$ :

$$
\alpha_{2}=-2 a h_{4}, \quad \alpha_{1}=0, \alpha_{0}=\frac{2}{3}\left(-a h_{2} \mp \sqrt{a^{2} h_{2}^{2}-3 a^{2} h_{0} h_{4}}\right), \omega=\frac{-3 a c \mp 4 a^{2} b \sqrt{a^{2}\left(h_{2}^{2}-3 h_{0} h_{4}\right)}}{4 a} .
$$

We then obtain

$$
v=-2 a h_{4} \varphi^{2}(\xi)-\frac{2}{3}\left(a h_{2} \pm \sqrt{a^{2} h_{2}^{2}-3 a^{2} h_{0} h_{4}}\right), \quad \omega=\frac{-3 a c \mp 4 a^{2} b \sqrt{a^{2}\left(h_{2}^{2}-3 h_{0} h_{4}\right)}}{4 a} .
$$

We substitute the general solutions [11] of Eq. (7) into Eqs. (32) and (33), respectively, and use Eq. (6), then two Jacobi elliptic function solutions of Jimbo-Miwa equation (1) are obtained. 
(i) If $h_{4}<0, h_{2}>0, h_{0}=\frac{1-m^{2}}{\left(2 m^{2}-1\right)^{2}}$, we obtain a Jacobi elliptic function solution

$u=\frac{2 a h_{2} m^{2}}{m^{2}+1} \int \operatorname{sn}^{2}\left(\sqrt{-\frac{h_{2}}{m^{2}+1}} \xi_{1}\right) d \xi_{1}-\frac{2}{3}\left(a h_{2} \pm \sqrt{a^{2} h_{2}^{2}-3 a^{2} h_{0} h_{4}}\right) \xi+d_{1}$

where $\xi=a x+b y+c z+\frac{3 a c \pm 4 a^{2} b \sqrt{a^{2}\left(h_{2}^{2}-3 h_{0} h_{4}\right)}}{4 a} t$.

(ii) If $h_{4}>0, h_{2}<0, h_{0}=\frac{h_{2}{ }^{2} m^{2}}{2 h_{4}\left(m^{2}+1\right)}$, we obtain another Jacobi elliptic function solution

$$
\begin{aligned}
& \quad u=\frac{2 a h_{2} m^{2}}{2 m^{2}-1} \int \operatorname{cn}^{2}\left(\sqrt{\frac{h_{2}}{2 m^{2}-1}} \xi_{1}\right) d \xi_{1}-\frac{2}{3}\left(a h_{2} \pm \sqrt{a^{2} h_{2}^{2}-3 a^{2} h_{0} h_{4}}\right) \xi+d_{1} \\
& \text { where } \xi=a x+b y+c z+\frac{3 a c \pm 4 a^{2} b \sqrt{a^{2}\left(h_{2}^{2}-3 h_{0} h_{4}\right)}}{4 a} t .
\end{aligned}
$$

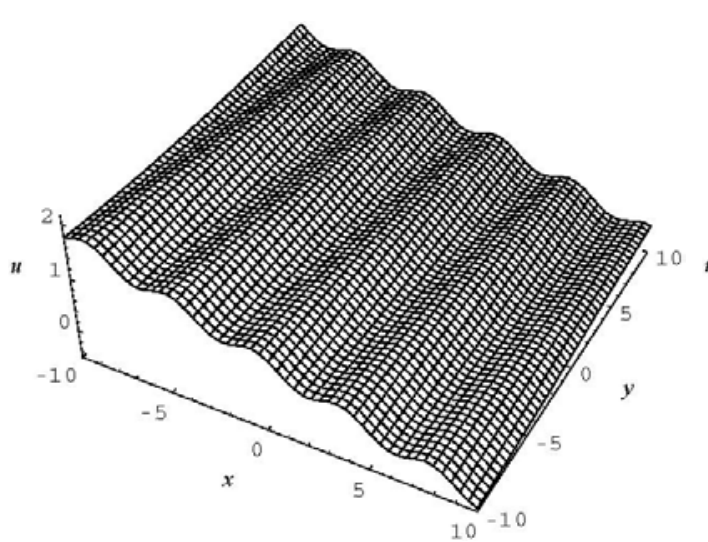

(a)

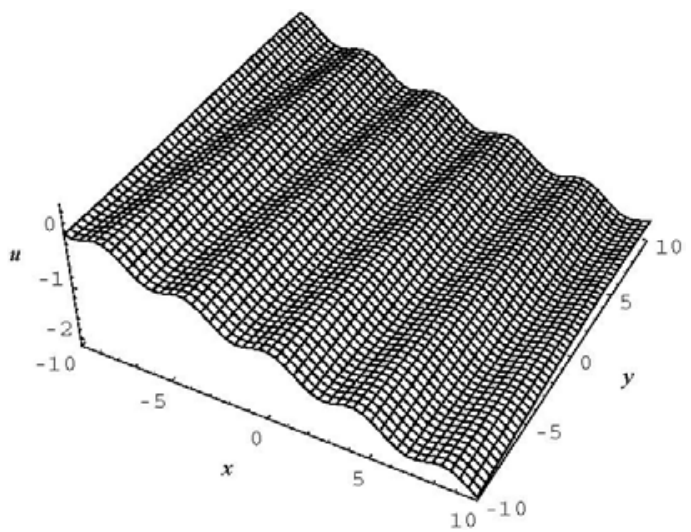

(c)

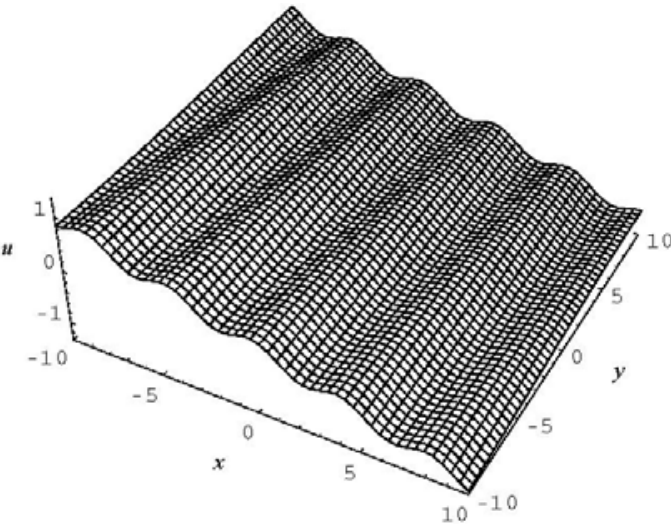

(b)

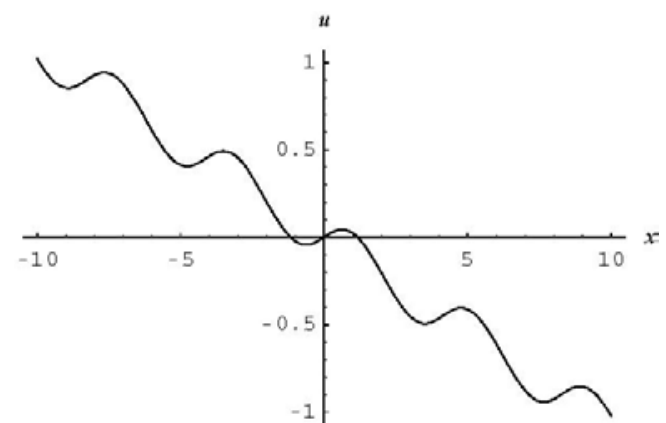

(d)

Fig. 1. Evolutionary plots of Jacobi doubly periodic solution (35) with (+) branch for parameters $a=1, b=0.1$, $c=2, d_{1}=0, h_{2}=-1, h_{4}=2, m=0.5, z=0$ at different times: (a) $t=-5$, (b) $t=0$, (c) $t=5$, (d) $y=0$, $t=0$.

In Fig. 1, the Jacobi doubly periodic solution (35) is shown. We can see from Fig. 1 that solution (35) has two remarkable evolutionary properties: (i) the wave propagation is skew; (ii) the amplitude enlarges along with the increasing time. It is the external linear function $\xi$ of solution (35)

$$
-\frac{2}{3}\left(a h_{2} \pm \sqrt{a^{2} h_{2}^{2}-3 a^{2} h_{0} h_{4}}\right) \xi+d_{1}=-1.66582 t+x+0.1 y
$$


which causes such evolutionary properties.

At the limit case $m \rightarrow 1$, the obtained Jacobi doubly periodic solutions (34) and (35) degenerate into the soliton solutions (19) and (29), respectively.

Case 3.4, when $h_{4}=h_{1}=h_{0}=0$ :

$$
\begin{aligned}
& \alpha_{2}=0, \quad \alpha_{1}=0, \quad \alpha_{0}=-\frac{1}{3} a h_{2}, \omega=\frac{-3 a c-a^{3} b h_{2}}{2 b}, \\
& \alpha_{2}=0, \quad \alpha_{1}=-\frac{1}{2} a h_{3}, \quad \alpha_{0}=0, \omega=\frac{-3 a c+a^{3} b h_{2}}{2 b} . \\
& \alpha_{2}=0, \quad \alpha_{1}=-\frac{1}{2} a h_{3}, \quad \alpha_{0}=-\frac{1}{3} a h_{2}, \quad \omega=\frac{-3 a c-a^{3} b h_{2}}{2 b} .
\end{aligned}
$$

Thus, we obtain

$$
\begin{aligned}
& v=-\frac{1}{3} a h_{2}, \quad \omega=\frac{-3 a c-a^{3} b h_{2}}{2 b}, \\
& v=-\frac{1}{2} a h_{3} \varphi, \quad \omega=\frac{-3 a c+a^{3} b h_{2}}{2 b}, \\
& v=-\frac{1}{2} a h_{3} \varphi-\frac{1}{3} a h_{2}, \omega=\frac{-3 a c-a^{3} b h_{2}}{2 b} .
\end{aligned}
$$

We substitute the general solutions [11] of Eq. (7) into Eqs. (40)-(43), respectively, and use Eq. (6), then three types of travelling wave solutions of Jimbo-Miwa equation (1) are obtained.

(i) If $h_{2}>0$, we obtain a rational solution and two kink-shaped soliton solutions

$$
\begin{aligned}
& u=-\frac{1}{3} a h_{2} \xi+d_{1}, \quad \xi=a x+b y+c z+\frac{3 a c+a^{3} b h_{2}}{2 b} t, \\
& u=\frac{a}{2} \sqrt{2 h_{2}} \tanh \left(\sqrt{\frac{h_{2}}{2}} \xi\right)+d_{1}, \quad \xi=a x+b y+c z+\frac{3 a c-a^{3} b h_{2}}{2 b} t, \\
& u=\frac{a}{2} \sqrt{2 h_{2}} \tanh \left(\sqrt{\frac{h_{2}}{2}} \xi\right)-\frac{1}{3} a h_{2} \xi+d_{1}, \quad \xi=a x+b y+c z+\frac{3 a c+a^{3} b h_{2}}{2 b} t .
\end{aligned}
$$

(ii) If $h_{2}<0$, we obtain the same rational solution (44) and two triangular periodic solutions

$$
\begin{aligned}
& u=-\frac{a}{2} \sqrt{-2 h_{2}} \tan \left(\sqrt{-\frac{h_{2}}{2}} \xi\right)+d_{1}, \quad \xi=a x+b y+c z+\frac{3 a c-a^{3} b h_{2}}{2 b} t, \\
& u=-\frac{a}{2} \sqrt{-2 h_{2}} \tan \left(\sqrt{-\frac{h_{2}}{2}} \xi\right)-\frac{1}{3} a h_{2} \xi+d_{1}, \quad \xi=a x+b y+c z+\frac{3 a c+a^{3} b h_{2}}{2 b} t .
\end{aligned}
$$

(iii) If $h_{2}=0$, we obtain two rational solutions

$$
\begin{aligned}
& u=-\frac{a}{2} \xi^{-1}+d_{1}, \quad \xi=a x+b y+c z+\frac{3 a c}{2 b} t, \\
& u=-\frac{a}{2} \xi^{-1}-\frac{1}{3} a h_{2} \xi+d_{1}, \quad \xi=a x+b y+c z+\frac{3 a c}{2 b} t .
\end{aligned}
$$

Case 3.5, when $h_{4}=h_{2}=0, h_{3}>0$ :

$$
\alpha_{2}=0, \quad \alpha_{1}=-\frac{a h_{3}}{2}, \quad \alpha_{0}= \pm \frac{\sqrt{3} a \sqrt{h_{1} h_{3}}}{6} i, \omega=\frac{-3 a c \pm \sqrt{3} a^{3} b \sqrt{h_{1} h_{3}} i}{2 b} .
$$


We then obtain

$$
v=-\frac{a h_{3}}{2} \varphi(\xi) \pm \frac{\sqrt{3} a \sqrt{h_{1} h_{3}}}{6} i, \omega=\frac{-3 a c \pm \sqrt{3} a^{3} b \sqrt{h_{1} h_{3}} i}{2 b},
$$

We substitute the general solutions [11] of Eq. (7) into Eq. (50), and use Eq. (6), then a Weierstrass elliptic function solution of Jimbo-Miwa equation (1) is obtained

$$
u=-\frac{a h_{3}}{2} \int \wp\left(\frac{\sqrt{h_{3}}}{2} \xi_{1}, g_{2}, g_{3}\right) d \xi_{1} \pm \frac{\sqrt{3} a \sqrt{h_{1} h_{3}} i}{6} \xi+d_{1}, \quad \xi=a x+b y+c z+\frac{3 a c \mp \sqrt{3} a^{3} b \sqrt{h_{1} h_{3}} i}{2 b} t,
$$

where $g_{2}=-\frac{4 h_{1}}{h_{3}}, g_{3}=-\frac{4 h_{0}}{h_{3}}$.

\section{Acknowledgements}

This work was supported by the PhD Start-up Funds of Liaoning Province of China (20141137) and Bohai University (bsqd2013025), the Natural Science Foundation of Educational Committee of Liaoning Province of China (L2012404), the Liaoning BaiQianWan Talents Program (2013921055) and the Natural Science Foundation of China (11371071).

\section{References}

[1] E.G. Fan: Phys. Lett. A Vol. 300 (2002), p. 243

[2] S. Zhang and T.C. Xia: Commun. Theor. Phys. Vol. 45 (2006), p. 985

[3] E.G. Fan and H.H. Dai: Comput. Phys. Commun. Vol. 153 (2003), p. 17

[4] S. Zhang, J.L. Tong and W. Wang: Comput. Math. Appl. Vol. 58 (2009), p. 2294

[5] E.G. Fan: J. Phys. A: Math. Gen. Vol. 36 (2003), p. 7009

[6] S. Zhang: Int. J. Comput. Math. Vol. 87 (2010), p. 885

[7] S. Zhang and B. Cai: Nonlinear Dyn. Vol. 78 (2014), p. 1593

[8] J.H. He, X.H. Wu: Chaos Soliton. Fract. Vol. 30 (2006), p. 700

[9] D. Wang, W.W. Sun, C.C. Kong and H.Q. Zhang: Appl. Math. Comput. Vol. 189 (2007), p. 878

[10]S. Zhang, J.H. Li and Y.Y. Zhou: Entropy Vol. 17 (2015), p. 3182

[11]E.G. Fan: Chaos Soliton. Fract. Vol. 16 (2003), p. 819

[12]S. Zhang and T.C. Xia: Appl. Math. Comput. Vol. 182 (2006), p. 1651

[13]S. Zhang and T.C. Xia: J. Phys. A: Math. Theor. Vol. 40 (2007), p. 227

[14]S. Zhang and T.C. Xia: Phys. Lett. A Vol. 356 (2006), p. 119

[15]S. Zhang and A.X. Peng: IAENG Int. J. Appl. Math. Vol. 44 (2014), p. 10 